\title{
Research on the Motor Drive Based Operating Mechanism for High Voltage Circuit Breaker
}

\author{
Yulong Huang ${ }^{1,}$, , Liucheng Hao ${ }^{2}$, Al-DweikatMohmmad ${ }^{1}$, Cheng \\ Tang $^{2}$,Shutong $\mathrm{Gao}^{2}$, Zhiyong Yang ${ }^{2}$ \\ ${ }^{1}$ State Key Lab. of Power System, Dept. of Electrical Engineering, Tsinghua University, Beijing \\ 100084, China \\ ${ }^{2}$ Pinggao Group Co., Ltd., Pingdingshan, Henan Province, 467001, China \\ ${ }^{a}$ E-mail: yulonghuang@tsinghua.edu.cn
}

Keyword: Circuit-Breakers; Motor drive; Simulink; Operating Mechanism

\begin{abstract}
This paper describes an operating mechanism driven by a three-phase permanent magnet synchronous motor (PMSM) for high voltage circuit breakers (HVCB). It analyzes the relationship between the optimal moving contact travel of HVCB and the PMSM torque, and proposes the main parameters and requirements of PMSM to be suitable for the optimal contact travel. The simulation model of the operating mechanism driven by PMSM was builtusing Matlab. It simulates and calculatesthe mechanism'sopening and closing operations characteristics. The voltage waveforms of stator currentsanalyzing, offers a helpful reference to compile the Digital Signal processing (DSP) programs. The performances of the opening and closing operationscould be improved by debugging the DSP programs in the control unit.
\end{abstract}

\section{Introduction}

High Voltage Circuit Breakers (HVCB) isthe most important switches apparatus in the electric power system. They are responsible forcontrol and protect of the power system. The operating mechanism is the basic device which controls the opening and closing operations of HVCB. At present, there are several conventional types of operating mechanisms which have been widely used, such as spring, hydraulic or pneumatic. Such mechanisms have many prominent merits, but also have some insurmountable limitations. These conventional mechanismsconstructedby complex frames, need periodic check-ups, highly costsoperating and maintenance,and they are quite difficult to be debugged. Additionally, they are especially incontrollable and non-detectable during the opening and closing operations.

To overcome such inherent limitations, and to improve the performance of conventional mechanisms, the operating mechanism driven by PMSMis introduced to meet the requirements. The proposed operating mechanism is based on power electronic and motor digital control technologies. This mechanism has quite a lot of good advantages: simple frames, manipulative opening and closing operations, wide range of uses and so on.

\section{Design of the operating mechanism}

The suggested operating mechanism consists of AC (or DC) power supply, energy buffer, converter unit, control unit, and the motor. Fig.1 (a) shows the System diagram of motor driven mechanism. The motor is controlled by an IGBT Converter, rotate speed and positions of the motor aremeasured using a fixed sensor on the rotating shaft. The travel of the moving contactor is measured by the position sensor.The control unit adopts DSPas the processing core, and according to the vector control of the motor, the control unit generates PWM signals to activate the IGBT Converter, and then drives the motor to implement the opening and closing operations of HVCB. 


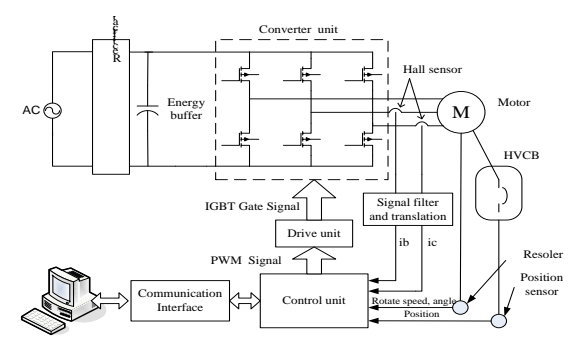

(a) System diagram

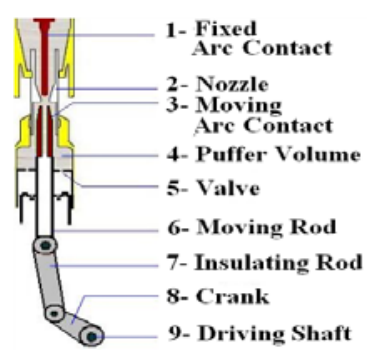

(b) actuating device

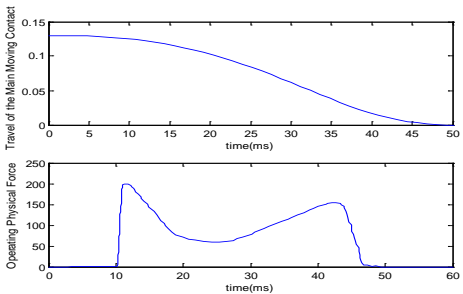

(c) Moving travel and driving force

Fig.1. Circuit breaker actuating device, where (b)

1- Fixed arc contact, 2-nozzle, 3-moving arc contact, 4-puffer volume, 5-valve, 6-Movingrod, 7-insulating rod, 8-crank, 9-driving shaft

\section{Simulation of the motor drive}

Fig.1 (b)shows the circuit breaker operating device.In the opening operation driven by the motor drivemechanism, the crank (8) rotates counterclockwise; pulling the insulating rod (7) down. This will quickly drives the moving arc contact (3) downwards. At the end ofthe opening operation, the motor applies a clockwise torque, as a buffering force, on thedriving shaft (9) to decelerate the crank (8) rotation. The moving arc contact(3) speed willsmoothly decrease and the mechanical impact will be limited.On the other hand, inthe closing operation: the crank (8) rotates clockwise, pushing the insulation rod (7) upwards. The moving contact (3) accelerates up toward the fixed contact. Using a pre-set calculations and control for speed and position, the motor then applies a reverse counterclockwise torque to decelerate the moving contact, thus to reduce the mechanical impact between the breaker's contacts.

In the initial period, SF6 circuitbreakers demand a great operating physical force to accelerate the moving parts and to compress SF6. This operating force makes the main moving contact reaches the demanded velocity requiredin the opening operation. Fig.3 (c) shows the curves of both the travel of the main moving contact and the operating physical force of HVCB.

The processes of the opening and closing operations are link motions made up of the moving parts. The velocity of the main moving contact can be calculated according to the travel as in Fig. 1 (b). Assumed that, the mass center of the main moving contact is at itscenter, and the mass centers of the connecting rod and the regulating lever are also at theircenters. Then the whole equivalent rotary (rotational) inertia $\mathrm{J}$ of the moving parts about the revolution (rotating) axis in part 9 shown in Fig.1 (b) are given as follows:

$$
J=J_{6}+J_{7}+J_{8}
$$

Of which,

$$
\begin{aligned}
& J_{6}=\frac{1}{3} m_{6} l_{6}^{2}+m_{6}\left(l_{7}^{2}+l_{8}^{2}+2 l_{7} l_{8} \cos \left(\theta_{2}-\theta_{3}\right)\right) \\
& J_{7}=\frac{1}{3} m_{7} l_{7}^{2}+m_{7}\left(l_{8}^{2}+l_{7} l_{8} \cos \left(\theta_{2}-\theta_{3}\right)\right) \\
& J_{8}=\frac{1}{3} m_{8} l_{8}^{2}
\end{aligned}
$$

Where m6, m7and $\mathrm{m} 8$ are themass quantities of the moving parts,16, 17 and 18 are the lengths,J6, $\mathrm{J} 7$ and $\mathrm{J} 8$ are the rotary rotational inertias about the revolution rotating axis.

In general, the whole equivalent rotary inertia will reach the maximum value at the initial position during the opening operation.

$$
J_{\max }=\left.\left(J_{6}+J_{7}+J_{8}\right)\right|_{\theta_{3}=\theta_{30}} ^{\theta_{2}=\theta_{20}}
$$

In the process ofopening operations, the kinetic energies of the moving parts are variable. Their equations are:

$$
\begin{aligned}
& E_{6}=\frac{1}{2} m_{6} V_{6}^{2}=\frac{1}{2} m_{6}\left(l_{8} \theta_{2} \sin \theta_{2}+l_{7} \ddot{\theta}_{3} \cos \theta_{3}\right)^{2} \\
& E_{7}=\frac{1}{2} m_{7} V_{7}^{2}=\frac{1}{2} m_{7}\left(V_{7 x}^{2}+V_{7 y}^{2}\right)
\end{aligned}
$$




$$
\begin{gathered}
=\frac{1}{2} m_{7}\left[\left(l_{8} \theta_{2} \sin \theta_{2}+\frac{1}{2} l_{7} \theta_{3} \sin \theta_{3}\right)^{2}+\left(l_{8} \theta_{2} \cos \theta_{2}+\frac{1}{2} l_{7} \theta_{3} \cos \theta_{3}\right)\right. \\
=\frac{1}{2} m_{7}\left[\left(l_{8} \theta\right)^{2}+\frac{1}{4}\left(l_{7} \theta_{3}\right)^{2}+l_{7} l_{8} \theta_{2} \theta_{3} \cos \left(\theta_{2}-\theta_{3}\right)\right] \\
E_{8}=\frac{1}{2} m_{8} V_{8}^{2}=\frac{1}{2} m_{8}\left(\frac{1}{2} l_{8} \theta_{2}\right)^{2}=\frac{1}{8} m_{8}\left(l_{8}{ }_{2}\right)^{2}
\end{gathered}
$$

WhereE6is the kinetic energy of the main moving contact, E7and E8 are the kinetic energies of the connecting rod and the regulating lever.

Considering the effects of the weight and the requirements of the operating physical force (considering the weight and the operating force requirements effects), the output power of the motor for driving the operating mechanism can be calculated (the operating mechanism driving motor output power can be calculated). The equation of the motor power is:

$$
P=\frac{\Delta\left(E_{6}+E_{7}+E_{8}\right)-\left(m_{6} g \Delta h_{6}+m_{7} g \Delta h_{7}+m_{8} g \Delta h_{8}\right)+O P F \cdot \Delta}{\Delta t}
$$

Whereh6, h7 and h8 are the fallen distances of m6, m7and m8, and OPF is the operating physical force.

For the analysis and calculations above, the torque affected(applied on) the regulating lever and the motor's power in the process of opening operation can be obtained. The curves of torque and the power of the drive motor can be seen inFig.2 (a). (The obtained/estimated drive motor torque and power are shown in Fig.2 (a)).

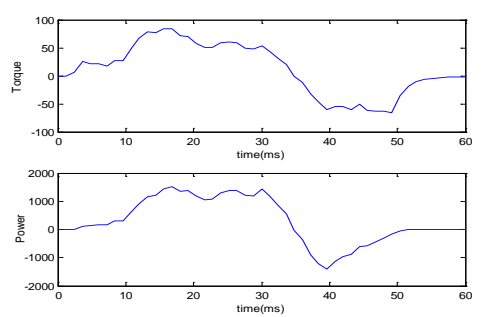

(a) the Torque and Power

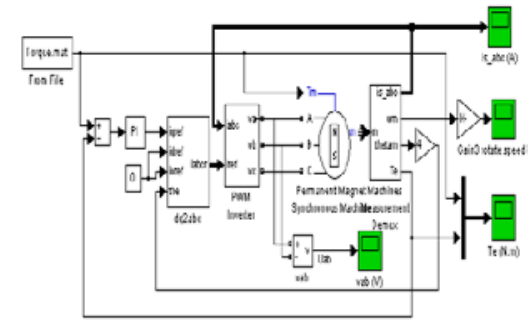

(b) the Simulation Model

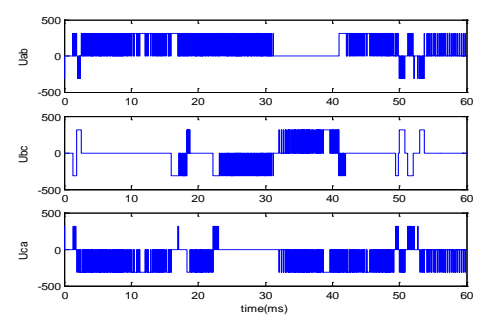

(c) the Voltage Waves of PMSM

Fig.2. the Simulation of actuating device

As could be noticed In Fig.2, the maximum instantaneous poweris $1500 \mathrm{~W}$, and the maximum instantaneous torque is $85 \mathrm{~N} \cdot \mathrm{m}$. As the time of the opening operation is about 40 to $60 \mathrm{~ms}$, the motor (rotates/operates) in the start-up phase. Therefore, the motor works in the transient state during the process of the opening operation. According as the maximum instantaneous torque can be 2 to 5 times than the nominal torque, and the maximum instantaneous power can be 2 to 4 times than the rated power, it is suitable to choose the motor that its nominal torque is 17 to $42.5 \mathrm{~N} \cdot \mathrm{m}$ and its rated power between 375 and $750 \mathrm{~W}$.

In addition,the chosen motor should have quite small moment of inertia, to carry out the opening and closing operations well.Since the whole equivalent rotary inertia of the moving parts is a relativelylarge (in the simulation model, $\mathrm{J}_{\max }=0.18 \mathrm{~kg} \cdot \mathrm{m}^{2}, \mathrm{~J}_{\min }=0.13 \mathrm{~kg} \cdot \mathrm{m}^{2}$ ). Based on the previous and by comparingwith othertypes of motors, PMSM (Permanent Magnet Synchronous Motor) ischosen to be the drive motor of the ( proposed HVCB operating )mechanism.

\section{Simulation Results}

The simulation model of the operating mechanism driven by the motor wasbuilt usingMatlab, shown in Fig.2.(a), the drive motor PMSM wasmanipulated by the vector control of id $=0$. The calculated torque wastaken as the input load of the PMSM. The deviation that is the error between the given torque and the feedback torque, passed through aPI adjuster,andwasput out as the reference $i_{\text {sqref }}$ current to control the PMSM torque. The $i_{\text {sqref }}$ and $i_{\text {sdref }}$ (equal to 0 ) currents 
passingthe Park and Clark transforms,turninto the current $i_{\text {abcref. }}$. Both $i_{\text {abcref }}$ and $i_{\text {sabc }}$ (the three-phase stator currents of the PMSM) as the comparative signals wereput into the PWM inverter, and then PWM signals weregenerated to control the PMSM. The parameters of PMSM in this simulation model were: $\mathrm{R}=2.875 \mathrm{ohm}, \mathrm{L}_{\mathrm{d}}=\mathrm{L}_{\mathrm{q}}=8.5 \mathrm{e}^{-3} \mathrm{H}, \varphi=0.25 \mathrm{~Wb}, \mathrm{P}=4$, andJ $=0.8 \mathrm{e}^{-3} \mathrm{~kg} \cdot \mathrm{m}^{2}$.

According to this simulation model, both the power output and the torque output of PMSM can be obtained. The curves of the power and the torque wereshown in Fig.2. (b), it is obvious that the power and torque outputs are suitable with the requirements of the operating mechanism driven by the motor.

Additionally, $\mathrm{U}_{\mathrm{ab}}, \mathrm{U}_{\mathrm{bc}}, \mathrm{U}_{\mathrm{ca}}$; the line voltages of PMSM can be obtained; their waves wereshownin Fig.2. (c), based on the line voltage waves, the IGBT switches sequences in the convert unit can be comprehended. The sequences offer a quite significant reference to compile the DSP program to control the PMSM rotation. The performances of the opening and closing operationscouldbe improved by debugging the DSP programs in the control unit.

\section{Conclusion}

This paper analyzes the relationships of the power and torque to operate the operating mechanism, and finally chooses PMSM to be the drive motor. By establishing the simulation model, the simulation results are accorded with the calculated. This means it is feasible to drive the operating mechanism of HVCB by PMSM in theory.

The operating mechanism power - torque characteristics were analyzed. Based on this characteristics, the PMSM was chosen to be the drive motor for the HVCB operating mechanism. The established simulation model results are in good agreement with the calculations. Such close agreement means to drive the HVCB operating mechanism by PMSM is feasible in theory.

\section{Acknowledgement}

In this paper, the research was sponsored by the National Natural Science Foundation of China under the referencenumber51377090, and Henan Pinggao Electric Co., Ltd, Pingdingshan, Henan Province, China

\section{References}

[1] A.Bosma, Franz-Josef Koerber, Roberto Cameroni, Richard Thomas, Motor Drive with Electronic Control for HVAC Circuit-Breakers,CIGRE Session 2002, Paris, France, pp. 13-203.

[2] A.Bosma, P-O. Thureson, A New Reliable Operating Mechanism for HVAC Circuit-Breakers, Transmission and Distribution conference and exposition. 2001 IEEE/PES, 28 Oct,-2 Nov. 2001, pp. 573-577.

[3] A.Bosma, Roberto Cameroni, Mark Blundell, Introducing a New Generation of Operating Mechanism for High Voltage AC Circuit-Breakers, Journal of Electrical \& Electronics Engineering, Australia, Vol. 21, no. 3, pp. 233-240, 2002

[4] P. Pillay, R. Krishnan, Modeling, simulation, and analysis ofpermanent-magnet motor drives, part 1: The permanent-magnet synchronousmotor drive, IEEE Transactions on Industrial electronics, vol.25, no. 2, pp. 256-273, 1989.

[5] Morimoto, S., Takeda, Y., Hatanaka, K., Tong, Y., Hirasa, T., Design and control system of inverter-driven permanent magnet synchronous motors for high torque operation, IEEE Transactions on Industry Applications, vol. 29, no. 6, 1993,pp. 1150 - 1155.

[6] Radwan T, Rahman M, Osheiba A, Dynamic analysis of a high performance permanent magnet synchronous motor drive, Proceedings of IEEE Canadian Conference of Electrical and Computer Engineering, Calgary, Alta. Canada, 26-29 May, 1996, pp. 611-614. 